\title{
Bayesian extreme modeling for non-stationary air quality data
}

\begin{abstract}
The aim of this paper is to model the non-stationary Generalized Extreme Value distribution with a focus on Bayesian approach. The location parameter is expressed in terms of linear trend over the time period while constant for both scale and shape parameters. This study also explores the informative and Jeffrey's prior towards the efficiency of the estimating procedure. Root Mean Square Error is then use for choosing the best prior. Metropolis Hasting for extreme algorithm will also briefly explained in this study. The model is applied to the air quality data for Johor state.
\end{abstract}

Keyword: Air-pollution; Bayesian analysis; Generalized extreme value distribution; Metropolis-Hastings algorithm; Non-stationary 